\title{
Production Rate Control of Unreliable Manufacturing Cell with Adjustable Capacity
}

\author{
ALI GHARBI $^{1}$, ADNENE HAJJI $^{2}$, KAREM GHOUIB $^{1}$ \\ ${ }^{1}$ Automated Production Engineering Department, Production Systems Design and Control \\ Laboratory, École de Technologie Supérieure, MonTRÉAL (Qc), Canada \\ ${ }^{2}$ Department of Operations and Decision Systems, UnIVERsité LAVAL, Québec (Qc), Canada
}

\begin{abstract}
This paper addresses the production control problem of an adjustable capacity unreliable manufacturing cell responding to a single product type demand. The manufacturing cell is composed of an unreliable machine, called the 'central machine'. Due to availability fluctuations, the central machine may fall short to meet the long term demand rate. In order to quickly adjust the production capacity and thus meet the demand, a reserve machine is called upon in support if the finished product inventory level drops below a specific threshold. Such a machine involves higher production costs compared with the central one. This paper aims to determine the optimal production control policy for the involved machines in order to minimize production, inventory and backlog costs over an infinite horizon. The paper proposes a continuous dynamic programming formulation of the problem and adopted a numerical scheme to solve the optimality conditions equations. The optimal production policy is shown to be described by a State Dependent Hedging Point Policy (SDHPP). To determine the optimal control policy parameters, an experimental approach based on design of experiment, simulation modeling, and response surface methodology is proposed. Several sensitivity analyses have been carried out and have shown the robust behavior of the developed policy facing expected variations of the system parameters. The results also show that the proposed SDHPP policy outperforms classical stand-by and parallel machines based control policies. The usefulness of the proposed approach is outlined for more complex situations where the system must deal with non-exponential failure and repair time distributions.
\end{abstract}

Keywords: Production rate control, Adjustable capacity, HJB equation, State Dependent Hedging Point Policy (SDHPP), Simulation. 


\section{Introduction}

Due to its importance for manufacturers, many research studies have been devoted to optimal production planning and control problems of stochastic manufacturing systems. Several approaches have been carried out aiming to address the problem for different classes of systems.

Let us start with the pioneering work of Kimemia and Gershwin (1983), which modelled the movement of parts as a continuous flow and suggested a feedback control of the flow rates of parts through a Flexible Manufacturing System (FMS). They showed that the optimal control policy, for the considered system, has a special structure called the Hedging Point Policy (HPP). Within such a policy, a non negative production surplus of part types, corresponding to the optimal inventory levels, is maintained during times of excess capacity availability to hedge against future capacity shortage caused by machine failures.

Akella and Kumar (1986) considered a single-machine, single-part-type system with linear holding and backlog cost. They assumed that the machine dynamic state is a birth-death process, i.e., the time between successive machine failures and repair times are exponentially distributed. They showed that the optimal production policy follow the aforementioned HPP. Bielecki and Kumar (1988) dealt with a similar problem with a long-run average cost objective. They demonstrated as well as in Kimemia and Gershwin (1983) that the HPP remains optimal.

With the assumptions of continuous failure-prone manufacturing systems and fixed maximal capacity, more general problems have been studied resulting in the characterization of optimal and sub-optimal production policies. Among others, Liberopoulos and $\mathrm{Hu}$ (1995) extended the Akella and Kumar proposal (1986) to the case of non-Markovian machine capacity process.

For the long-run average cost and multiple machine states, Sharifnia (1988) extended the proposal by Bielecki and Kumar (1988) and showed that the optimal production policy has a Multiple Hedging Point (MHP) structure. Liberopoulos and Caramanis (1994) demonstrated that the Sharifnia's approach applies when the machine transition rates depend on the production rates. Within the class of MHP policy, Gharbi et al. (2008) showed that the policy guarantees better performance than the HPP to control production rates for remanufacturing systems subject to stochastic unplanned demand. In the same direction the MHP policy was employed by Kenne et al. (2003) to control the corrective maintenance rates in a multi-machine, multi-product, unreliable manufacturing system. The considered policy is governed by two hedging points 
designed to readjust the corrective maintenance rate which depends on the available inventory levels. A staircase structure policy or multiple hedging point age-dependent policy has been shown optimal (minimizing an overall cost function, including inventory holdings, lost sales, preventive and corrective maintenance costs) by kenne et al. (2007). From a practical point of view, Pellerin et al. (2007) have shown that this class of policy can also be integrated in an ERP system (i.e., Enterprise Resource Planning) to control production and corrective maintenance rates for stochastic manufacturing systems governed by an inspection process. It is interesting to note that when considering a given manufacturing system within its external environment (i.e., supply chain) this class of policy is also very efficient. In fact, to determine a control policy for both the supply and remanufacturing activities that minimizes the average repair/replacement, acquisition and inventory/shortage total cost over an infinite horizon, Berthaut et al. (2009) have proposed a suboptimal joint remanufacturing and supply control policy. Such a policy is composed of a MHP policy for the remanufacturing stage and an (s, Q) policy for the spare parts supply. Within such a policy an economic lot of raw material is ordered when the upstream inventory level riches s. Furthermore, it has been shown that the proposed MHP policy leads to a significant cost reduction as compared to a combination of a HPP and an (s, Q) policy. In the same direction, Hajji et al. (2009) have developed a complete strategy for production and replenishment activities which combines a modified (s, Q) and MHP policies. For an extended survey in optimal control of stochastic manufacturing systems, we refer the reader to Gershwin (2000), Sethi et al. (2002), and Zanoni et al. (2006).

For the class of manufacturing systems under consideration where the existing machine capacity falls short to meet the demand due to failure events and reparation activities, the problem remains open. This class of manufacturing systems can be considered as a special case of one called the Reconfigurable Manufacturing System (RMS) (Koren et al. (1999)). In this case, the production planning problem has never been addressed from a stochastic operational point of view. A RMS is defined as a system designed for rapid change in its structure, as well as its hardware and software components, in order to quickly adjust its production capacity and functionality within a part family in response to sudden market changes or intrinsic system change (Koren et al. (1999); Koren and Ulsoy (2002)). This class of manufacturing systems can also be considered as a decision making problem to adjust capacity by machine replacement when the system faces imperfect repairs for example (Dehayem Nodem et al. (2009)). In this paper, the problem is addressed from an operational point of view to keep the decision for capacity adjustment following, in a continuous manner, the operational decisions. 
The paper's main contribution lies in the development of a production control policy for a monoproduct manufacturing cell composed of a single unreliable workstation called the 'Central Machine'. This manufacturing cell is a part of a Cellular Manufacturing System (CMS) composed of several other cells (Askin and Standridge (1993), Wu et al. (2007)). The considered cell capacity can be adjustable by considering a reserve machine incurring higher production costs when it is used. Usually, stand-by machines are called upon in support when the central machine breaks down and until reparation ends (Freiheit et al. (2004), Jardine and Tsang (2005)). However, the resultant control policy, called the State Dependent Hedging Point Policy (SDHPP), is more realistic, flexible, and useful in this context. Under the SDHPP policy, the reserve machine is called upon in support to production only if the finished product inventory level drops below a specific threshold. The SDHPP policy depends not only on the central machine state but also on the state of finished product inventory. The paper's contribution is further illustrated by the robust behaviour of the SDHPP facing expected variations of the system parameters. A simulation-based experimental design approach is combined with the control theory to develop a systematic control approach to achieve a close approximation of the optimal production policy. Extensions to cover more complex systems, for which the failure and repair times are non-exponentially distributed, are also outlined.

This paper is organized as follows: Section 2 presents the statement of the optimal production problem. The numerical approach and the related control policy are presented in section 3 . Sections 4 and 5 describe the combined control approach and the simulation model. Section 6 outlines the experimental results and the usefulness of the proposed control policy. A comparison study between the proposed SDHPP policy and control policies based on classical stand-by and parallel machines is carried out in section 7. An Extension to manufacturing system with nonexponential failure and repair time distributions is presented in section 8 . The paper is concluded in section 9 .

\section{Problem Statement}

The manufacturing system under study consists of an unreliable central machine $M_{1}$ capable of producing one part type $P$ (Figure 1). Due to randomness of its own availability (i.e., failure events and repair activities), $M_{1}$ capacity falls short to meet the demand $d$. Therefore, a reserve machine $M_{2}$ is called to ensure a long term satisfaction of the demand. Such an action cannot be realised in a continuous manner due to the higher production costs of $M_{2}$ compared to those 
of $M_{1}$. In fact, and founded on group technology and CMS concepts (Burbidge (1992), Andrés et al. (2005), Yang and Yang (2008)), the central machine, which is dedicated to producing product $\mathrm{P}$, is selected based on several performance criteria; especially, its throughput rate and production cost. Due to higher cost incurred by the reserve machine $\mathrm{M}_{2}$; this latest is stopped as soon as machine $M_{1}$ is restored to operational state and a certain inventory level is reached to hedge against demand shortages. Figure 1 illustrates the system under study, its dynamic behaviour, and the associated costs to be minimized.

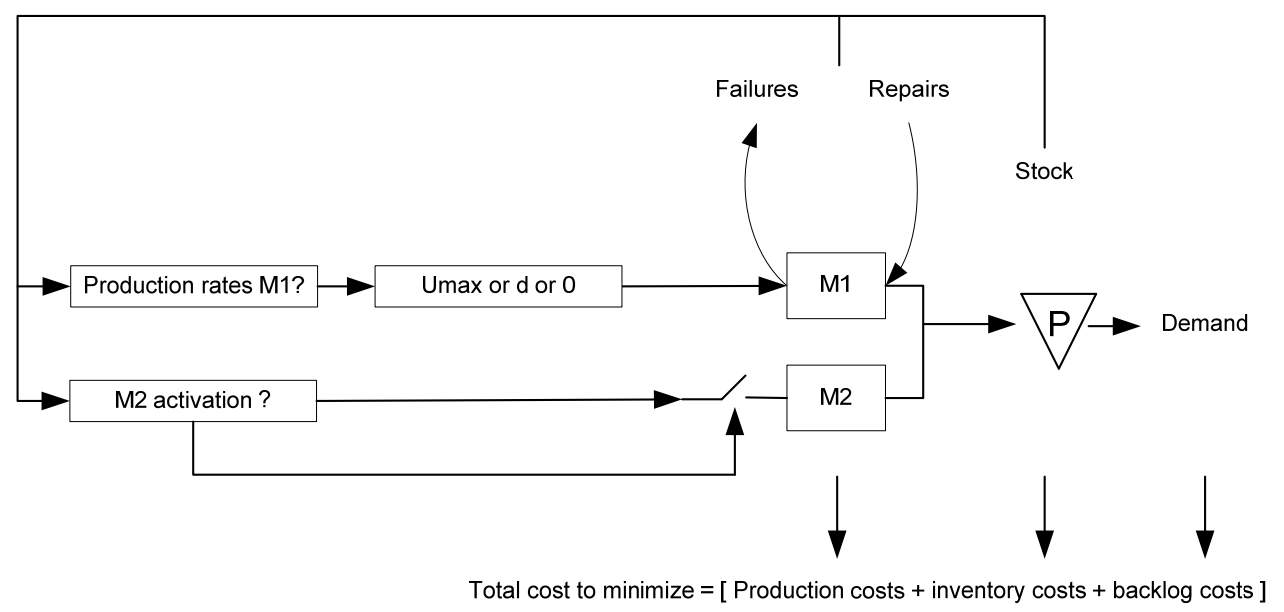

Figure 1: Structure of the manufacturing system under study

To be processed, a part type $P$ requires an average processing time equal to $\mathrm{p}>0$. Also, product $P$ has an average time between orders equal to $1 / d$, assumed to be constant. For the considered manufacturing system, $x(t), u_{1}(t)$ and $u_{2}(t)$ denote the inventory/backlog level of $P$, the central machine and the reserve machine production rates, respectively, at time $t$.

For any specific time t, the state of the system has two components: a continuous component denoted $x(t)$ describing the cumulative surplus level, and a discrete component denoted $\alpha(t)$ describing the central machine state. The central machine state $\alpha(t)$ is a continuous time discrete space stochastic process taking value 0 if machine $M_{1}$ is under repair and 1 if it is operational. Hence, the reconfigurable manufacturing system dynamic behaviour can be modeled by the state variables $(x(t), \alpha(t))$ where $x(t) \in \mathscr{R}, \alpha(t) \in \mathrm{M}=\{0,1\}$.

The dynamic behaviour of the product surplus is given by the following differential equation: 


$$
\dot{x}(t)=u_{1}(t, \alpha)+u_{2}(t)-d, \quad x(0)=x
$$

where $x$ denotes the initial surplus level.

The central machine uptimes and downtimes are assumed to be exponentially distributed with failure and repair rates equal to $p_{1}$ and $r_{1}$, respectively. The machine state evolves according to a continuous-time Markov process with states in $\mathrm{M}$ and with a generator matrix $Q$ such that: $Q=\left\{q_{\alpha \beta}\right\}$, where $q_{\alpha \beta}$ denotes the transition rate from state $\alpha$ to $\beta, q_{\alpha \beta} \geq 0$, and $q_{\alpha \alpha}=-\sum_{\beta \neq \alpha} q_{\alpha \beta}, \alpha, \beta \in \mathrm{M}$. The transition rate matrix $\boldsymbol{Q}$ is expressed as follows:

$$
Q=\left|\begin{array}{cc}
-q_{01} & q_{01} \\
q_{10} & -q_{10}
\end{array}\right| \text { where } q_{10}=p_{1} \text { and } q_{01}=r_{1} \text {. }
$$

The production rates at any given time must satisfy the capacity constraint of the machines given by equation (3); where $u_{1}^{\max }$ and $u_{2}^{\max }$ denote the maximum production rates of machines $M_{1}$ and $M_{2}$, respectively.

$$
0 \leq u_{i}(t) \leq u_{i}^{\max }, i=1,2
$$

Due to the random availability of the central machine, its maximum production rate falls short to meet the demand. Given the stochastic process governing machine $M_{1}$, its steady state availability can be calculated as follows:

$$
U T R_{M_{1}}=\frac{r_{1}}{r_{1}+p_{1}}
$$

Thus, the availability constraint of machine $M_{1}$ is given by the following equation:

$$
u_{1}^{\max } \times \frac{r_{1}}{r_{1}+p_{1}}<d
$$


Considering the available capacity of machine $M_{2}$, the whole capacity of the system can meet the demand, and the overall capacity constraint is given by:

$$
u_{1}^{\max } \times \frac{r_{1}}{r_{1}+p_{1}}+u_{2}^{\max } \geq d
$$

For each $\alpha \in \mathrm{M}$, the feasible production rate (or capacity) set is given by:

$$
\Gamma(\alpha)=\left\{\left(u_{1}, u_{2}\right) \geq 0,0 \leq u_{i}(t) \leq u_{i}^{\max }, i=1,2, u_{1}^{\max } \times \frac{r_{1}}{r_{1}+p_{1}}+u_{2}^{\max } \geq d\right\}
$$

The decision variables of the control policy are the production rates $\left(u_{1}(),. u_{2}().\right)$ of the central machine $M_{1}$ and the reserve machine $M_{2}$. Intrinsic machines properties stipulate different speed over which a given machine can operate the product. Machine $M_{1}$ can be shut down (i.e, $u_{1}(t)=0$ ), operate under the demand rate (i.e, $u_{1}(t)=d$ ), or operate under its maximum production rate (i.e, $u_{1}(t)=u_{1}^{\max }$ ). Concerning machine $M_{2}$, it can be shut down (i.e, $u_{2}(t)=0$ ), or operate under its maximum production rate (i.e, $u_{2}(t)=u_{2}^{\max }$ ). The decision made by the manager to choose which machine to operate and under which production rate is strongly conditioned by the involved production costs. Let $C_{M_{1}}^{1}, C_{M_{1}}^{2}$ and $C_{M_{2}}$ denote the production cost of machine $M_{1}$ under the demand production rate, the production cost of machine $M_{1}$ under the maximum production rate and the production cost of machine $M_{2}$, respectively. These costs must satisfy constraint (8) stating that machine $M_{1}$ production costs are proportional to its speed and that operating the reserve machine $M_{2}$ is much more costly.

$$
0<C_{M_{1}}^{1}<C_{M_{1}}^{2}<<C_{M_{2}}
$$

The inventory, backlog and production cost function $g($.$) is given by the following equation:$ 


$$
\begin{aligned}
& g\left(x(t), u_{1}(t), u_{2}(t)\right)=C^{+} x^{+}+C^{-} x^{-}+C_{M_{1}}^{l} \times u_{1}(t)+C_{M_{2}} \times u_{2}(t) \\
& l=\left\{\begin{array}{lll}
1 & \text { if } & u_{1}(t)=d \\
2 & \text { if } & u_{1}(t)=u_{1}^{\max }
\end{array}\right.
\end{aligned}
$$

where $x^{+}=\max (0, x), x^{-}=\max (-x, 0), C^{-}$: backlog cost and $C^{+}$: inventory cost.

The discounted total cost $J($.) can be defined by the following expression:

$$
J\left(x, \alpha, u_{1}, u_{2}\right)=E\left[\int_{0}^{\infty} e^{-\rho . t} g\left(x, u_{1}, u_{2}\right) \cdot d t\right]
$$

where $\rho$ denotes the discounted rate of the incurred cost and $\mathrm{E}\left[\cdot \mid x_{0}, \alpha_{0}\right]$ is the expectation operator conditional on initial condition.

Let $A$ denote the set of admissible decisions $\left(u_{1}(),. u_{2}().\right)$. The considered production planning problem consists in finding an admissible decision or control policy $\left(u_{1}(),. u_{2}().\right)$ that minimizes the discounted total cost $J($.$) (Eq. 10), subject to constraints (1) to (9). Such a feedback control$ policy, as illustrated in Figure 1, determines the production rates as a function of the surplus level $x$ and the state of the system $\alpha$.

While producing the part type $P$, the corresponding value function $v($.$) can be given by:$

$$
v(x, \alpha)=\inf _{\left(u_{1}, u_{2}\right) \in A} J\left(x, \alpha, u_{1}, u_{2}\right) \quad \forall x \in \mathscr{R}, \alpha \in M
$$

One can show that the value function $v(x, \alpha)$ is locally Lipschitz, and is the unique viscosity solution to the following HJB equation.

$$
\min _{\left(u_{1}, u_{2}\right) \in \Gamma(\alpha)}\left[\left(u_{1}+u_{2}-d\right)(v)_{x}(x, \alpha)+g\left(x, u_{1}, u_{2}\right)+Q . v(x, .)(\alpha)\right]-\rho v(x, \alpha)=0
$$

where $(v)_{x}($.$) denotes the gradients of v($.$) with respect to \boldsymbol{x}$. 
The production policy that we are seeking is obtained when the value function is known. While it's very complex if not impossible to analytically solve the HJB equations (12), the next section proposes a numerical method to obtain the approximation of the value function and the associated control policy.

\section{Numerical Approach and Optimal Control Policy}

In order to approximate the solution of the HJB equations (12) corresponding to the stochastic optimal control problem, and to solve the corresponding optimality conditions, a numerical method based on Kushner and Dupuis (1992) approach is proposed. The basic idea consists in using an approximation scheme for the gradient of the value function $(v)_{X}($.$) .$

\subsection{Numerical optimality conditions}

Let $h$ denotes the length of the finite difference interval of the variable $x$. Using the finite difference approximation, $v(x, \alpha)$ could be given by $v^{h}(x, \alpha)$, and the gradient $(v)_{x}(x, \alpha)$ by:

$$
(v)_{x}(x, \alpha)= \begin{cases}\frac{1}{h}\left(v^{h}(x+h)-v_{i}^{h}(x)\right) & \text { if } u_{1}+u_{2}-d \geq 0 \\ \frac{1}{h}\left(v^{h}(x)-v^{h}(x-h)\right) & \text { if } u_{1}+u_{2}-d<0\end{cases}
$$

Also, we could see that:

$$
\begin{aligned}
\left(u_{1}+u_{2}-d\right)(v)_{x}(x, \alpha)= & \frac{\left|u_{1}+u_{2}-d\right|}{h} v^{h}(x+h) \operatorname{Ind}\left\{u_{1}+u_{2}-d \geq 0\right\} \\
& +\frac{\left|u_{1}+u_{2}-d\right|}{h} v^{h}(x-h) \operatorname{Ind}\left\{u_{1}+u_{2}-d<0\right\} \\
& -\frac{\left|u_{1}+u_{2}-d\right|}{h} v^{h}(x)
\end{aligned}
$$

Using this approximation, the HJB equations (12) can be expressed in terms of $v^{h}(x, \alpha)$, as shown in equation (15). 


$$
v^{h}(x, \alpha)=\min _{\left(u_{1}, u_{2}\right) \in \Gamma(\alpha)}\left[\begin{array}{l}
\left(\rho+\left|q_{\alpha \alpha}\right|+\frac{\left|u_{1}+u_{2}-d\right|}{h}\right)^{-1} \\
\left(\begin{array}{l}
\frac{\left|u_{1}+u_{2}-d\right|}{h} \times v^{h}(x+h) \times \operatorname{Ind}\left(u_{1}+u_{2}-d \geq 0\right)+ \\
\frac{\left|u_{1}+u_{2}-d\right|}{h} \times v^{h}(x-h) \times \operatorname{Ind}\left(u_{1}+u_{2}-d<0\right) \\
+g\left(x, u_{1}, u_{2}\right)+\sum_{\beta \neq \alpha} q_{\alpha \beta} \cdot v^{h}(x, \beta)
\end{array}\right)
\end{array}\right]
$$

The solution of the numerical approximation of $v(x, \alpha)$ may be obtained by either successive approximation or policy improvement techniques (Kushner and Dupuis (1992)).

\subsection{Numerical results}

The implementation of the approximation technique requires the use of a finite grid denoted by $G_{h}$. The considered computation domain $\mathrm{D}$ is given by:

$$
\mathrm{D}=\{x:-5 \leq x \leq 5, h=0.1\}
$$

The numerical results used to characterize the optimal production policy are analyzed in this section within a basic case. Table 1, shows the operational and cost parameters of the considered system.

Table 1: Data parameters

\begin{tabular}{|c|c|c|c|c|c|c|}
\hline Parameters & $\left(C^{+} ; C^{-}\right)$ & $\left(C_{M_{1}}^{1} ; C_{M_{1}}^{2} ; C_{M_{2}}\right)$ & $\left(p_{1} ; r_{1}\right)$ & $\left(u_{1}^{\max } ; u_{2}^{\max }\right)$ & $d$ & $\rho$ \\
\hline Values & $(5 ; 50)$ & $(3 ; 10 ; 60)$ & $(0.04 ; 0.15)$ & $(0.25 ; 0.05)$ & 0.21 & 0.01 \\
\hline
\end{tabular}

It follows from numerical results that the optimal production policy has a multiple hedging point structure, which we call the 'State Dependent Hedging Point Policy' (SDHPP) (Fig. 2); where $Z_{1}$ and $Z_{2}$ define the two thresholds governing the policy. 

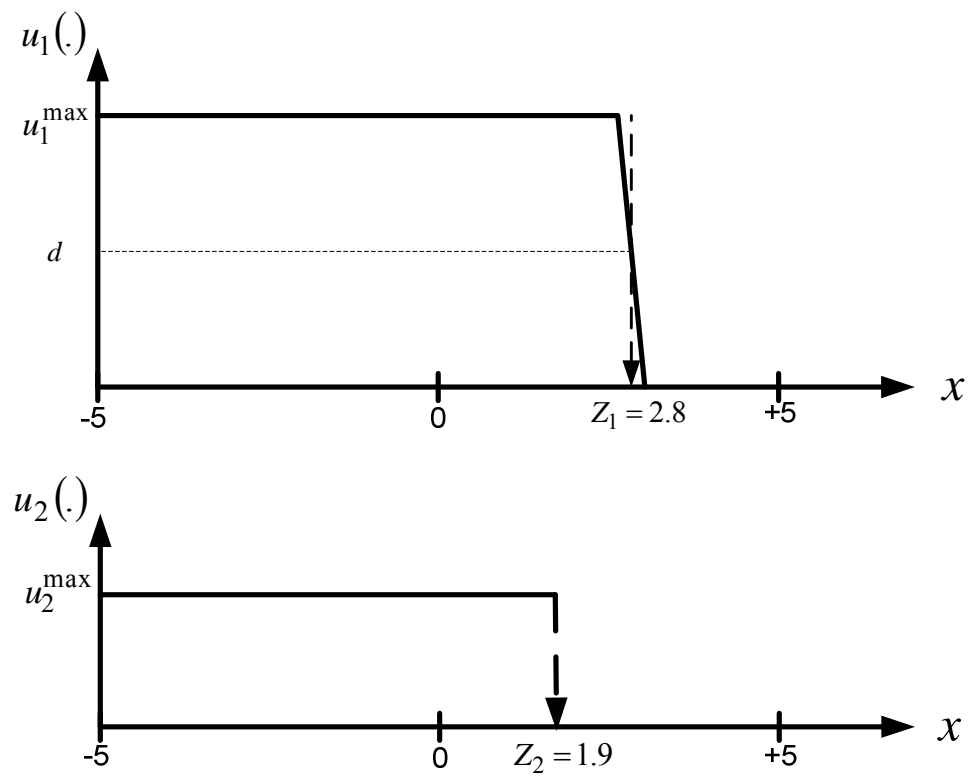

Figure 2: Production control policy

The results obtained show that the production control policy for the two machines is defined by two hedging levels. According to a HPP policy, the central machine $M_{1}$ must produce at its maximum production rate as long as the stock level $x(t)$ is lower than the hedging level $Z_{1}=2.8$. When $x(t)$ reaches $Z_{1}$, the production rate must be fixed to the demand rate. Above $Z_{1}$ the machine is shut down and the production rate is fixed to zero. The reserve machine $M_{2}$ is also governed by a class of a HPP policy called bang-bang policy but according to a different hedging level denoted $Z_{2}$. Thus $M_{2}$ must produce at its maximum production rate as long as the stock level $x(t)$ is lower or equal to the hedging level $Z_{2}=1.9$. Above $Z_{2}$ the machine is shut down and its production rate is fixed to zero.

The obtained policy makes sense since the production cost of the reserve machine is higher than that of the central machine. Therefore, in the negative area of the stock (i.e., shortage of stock) the random availability of the central machine makes it impossible for the system to reach a safety stock level to hedge against future breakdowns and the support of $M_{2}$ is necessary to achieve such a goal. This support cannot be maintained continuously and is kept only for a given thresh of the stock space. In this case, the considered data parameters have led to a positive hedging level $Z_{2}$ which is lower than $Z_{1}$. This result also makes sense since the lower production cost of the central machine makes it the one to be used most often in reaching the security stock level. 
However, the thresh limit under which the reserve machine must be called in support is obviously fluctuating and is defined according to a compromise of the production costs and rates. This issue is detailed in the next section.

Figure 3 illustrates the dynamic behaviour of the production control policy over time. As explained previously, three areas can be defined:

- Area 1: under the hedging level $Z_{2}$, the two machines must produce according to their maximum production rates $\left(u_{1}^{\max } \times \alpha+u_{2}^{\max }\right)$.

- Area2: between the hedging levels $Z_{1}$ and $Z_{2}$, the central machine $M_{1}$ must produce according to its maximum production rate. Machine $M_{2}$ is shut down.

- Area 3: when the stock level reaches $Z_{1}$, the central machine $M_{1}$ must produce according to the demand rate.

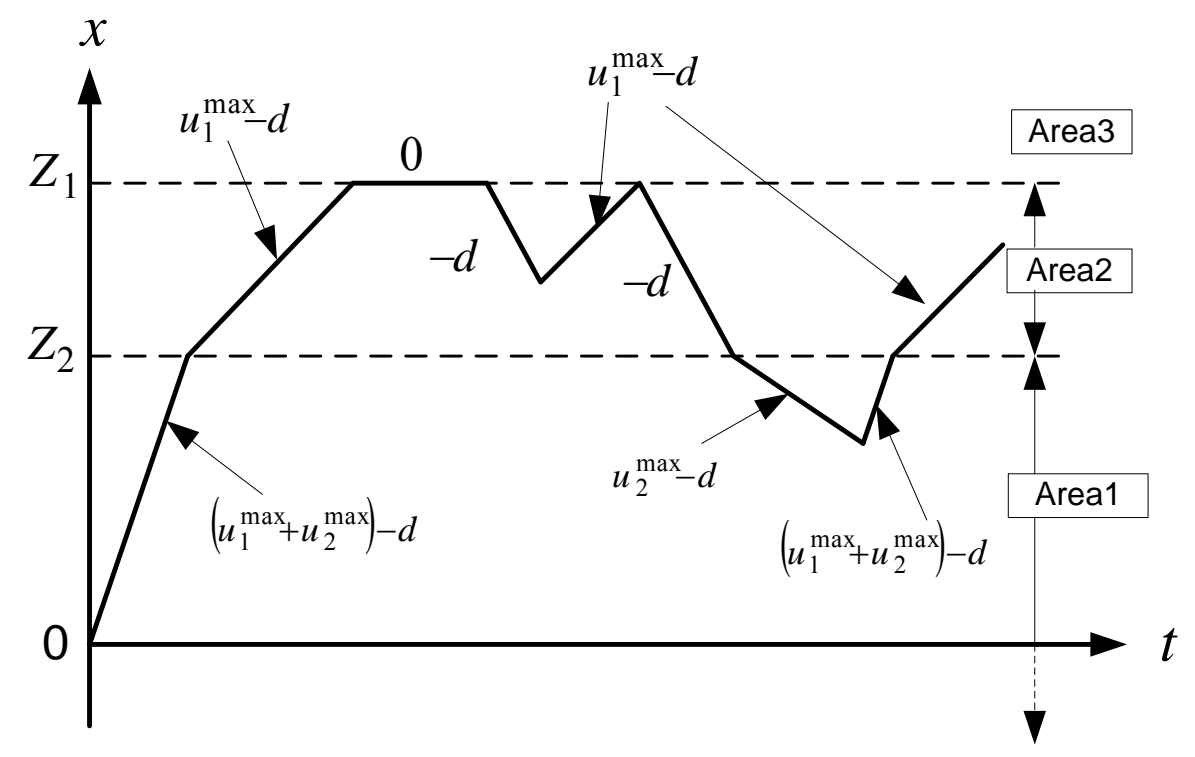

Figure 3: dynamic behaviour of the stock over time

In the next section, sensitivity analysis and several experimentations are conducted to ensure that the structure of the obtained policy is maintained and can be considered as a generalized policy for the general problem under study. 


\subsection{Sensitivity analysis: Parameterized control policy}

To illustrate the effect of system parameters changing on the proposed SDHPP policy, a sensitivity analysis has been performed (Table 2). Five sets of parameter variations have been conducted:

- Set I consists in 3 cases with backlog cost variation.

- Set II consists in 3 cases with inventory cost variation

- Set III and IV consist in 6 cases with machine $M_{2}$ production cost variation.

- Set $\mathrm{V}$ consists in 3 cases with machine $M_{2}$ production rate variation.

Sets I and II results show that when the backlog cost rises, the value of the hedging thresholds increase accordingly to ensure the availability of enough stocks to hedge against future backlogs. Moreover, when the inventory cost increases, the values of the hedging thresholds decrease to confine the stock accumulation.

Table 2: Data parameters for the sensitivity analysis cases

\begin{tabular}{|c|c|c|c|c|c|c|}
\hline & Cases & $C^{+}$ & $C^{-}$ & $C_{M_{2}}$ & $u_{2}^{\max }$ & $\left(Z_{1}^{*}, Z_{2}^{*}\right)$ \\
\hline \multirow{4}{*}{ Set I } & Basic & 5 & 50 & 60 & 0.05 & $(2.8 ; 1.9)$ \\
\cline { 2 - 7 } & 1 & 5 & 60 & 60 & 0.05 & $(3 ; 2.2)$ \\
\cline { 2 - 7 } & 2 & 5 & 70 & 60 & 0.05 & $(3.3 ; 2.4)$ \\
\hline \multirow{4}{*}{ Set II } & Basic & 5 & 50 & 60 & 0.05 & $(2.8 ; 1.9)$ \\
\cline { 2 - 7 } & 1 & 6 & 50 & 60 & 0.05 & $(2.4 ; 1.7)$ \\
\cline { 2 - 7 } Set III & 2 & 7 & 50 & 60 & 0.05 & $(2.2 ; 1.5)$ \\
\cline { 2 - 7 } & 1 & 5 & 50 & 80 & 0.05 & $(2.9 ; 1.7)$ \\
\hline & 2 & 5 & 50 & 100 & 0.05 & $(3.1 ; 1.5)$ \\
\hline \multirow{3}{*}{ Set IV } & 1 & 5 & 50 & 55 & 0.02 & $(2.7 ; 2)$ \\
\cline { 2 - 7 } & 2 & 5 & 50 & 50 & 0.02 & $(2.7 ; 2.2)$ \\
\cline { 2 - 7 } & Basic & 5 & 50 & 60 & 0.05 & $(2.8 ; 1.9)$ \\
\hline \multirow{3}{*}{ Set V } & 1 & 5 & 50 & 60 & 0.06 & $(2.4 ; 1.5)$ \\
\cline { 2 - 7 } & 2 & 5 & 50 & 60 & 0.07 & $(2.2 ; 1.1)$ \\
\cline { 2 - 7 } & & & & & & \\
\hline
\end{tabular}


Set III results show that when the production cost of the reserve machine increases the hedging level $Z_{2}$ decreases. Additional experimentations have been conducted with a higher $C_{M_{2}}$ and showed that the level of $Z_{2}$ still decreasing to reach zero. This result makes sense since a very costly reserve machine should be called in support only for critical situations (i.e., negative stock level). Set IV results confirm this observation and show that when the production cost of the reserve machine decreases the hedging level $Z_{2}$ increases and the machine is called to support the central one more often.

It is interesting also to observe the results of Set V showing that with a higher production rate of the reserve machine the two hedging levels decrease. This observation show that since the available capacity can ensure a rapid return to normal after machine failures, the need for high safety stock levels is lower.

From the above analysis, it clearly appears that the results obtained make sense, and that the structure of the policy defined by the 2 parameters $\left(Z_{1}\right.$ and $\left.Z_{2}\right)$ is always maintained. This allows the development of a parameterized production control policy defined by the following equations:

$$
\begin{aligned}
& u_{1}(.)=\left\{\begin{array}{ccc}
u_{1}^{\max } & \text { if } & x<Z_{1} \\
d & \text { if } & x=Z_{1} \\
0 & \text { if } & x>Z_{1}
\end{array}\right. \\
& u_{2}(.)=\left\{\begin{array}{ccc}
u_{2}^{\max } & \text { if } & x \leq Z_{2} \\
0 & \text { if } & x>Z_{2}
\end{array}\right.
\end{aligned}
$$

with the following constraint:

$$
Z_{2} \leq Z_{1} \text { and } 0 \leq Z_{1}
$$

The state dependent hedging point policy presented by equations (16) to (18) is completely defined for given values of $Z_{i}(i=1,2)$, called here design factors.

The numerical methods provide the near-optimal structure of the control policy. However, implementation difficulties and irregularities in the regions boundaries make the approximation of the control parameters challenging. Furthermore, the accuracy of the values of the minimum value function and of the control parameters obtained with the numerical methods depends on how fine the step of the grid is (Kenne et al. 2003). A satisfactory approximation would be too 
time-consuming to be applied to the operational level, which is one of our objectives. Consequently, we propose an alternative approach based on simulation in order to approximate the optimal control parameters and the associated cost. The simulation based approach is more flexible and more suited to thoroughly compare and extend the control policies in a wide range of time and cost configurations. The next sections are aimed at developing a systematic approach for determining optimal values of $Z_{i}(i=1,2)$ and to outline possible extensions to cover more realistic systems where the central machine is facing non-exponential failure and repair time distributions.

\section{Resolution approach}

In control theory, the results of traditional optimization methods are sometimes insufficient to reach a comfortable level of performance. Optimal solutions are difficult to calculate and are often approximated by numerical methods under strict conditions. This fact limits the usefulness of the obtained solution for more realistic cases.

To improve these methods, we have proposed an approach combining the analytical models with the descriptive capacities of simulation models, with experimental design, and response surface methodology techniques (Gharbi et al. (2006)). To quantify the policy, which structure is given by analytical model, simulation model are combined with experimental design and response surface methodology to estimate the optimal values of the policy's parameters. In the case of nonexponential failure rate and repair time distributions, the quantification parameters is also possible with the help of the simulation model, which can easily take into account the nature of any probability distributions. The corresponding output is then given by the simulation model (i.e., cost) which affects the response surface model.

This approach, applied to the problem presented in this paper, consists on the following sequential steps:

Step 1. The Structure of near-optimal control policy given by equations (16) to (18) is obtained, based on the numerical approach developed in section 3 . The Control factors $Z_{i}(i=1,2)$ fully describe the control policy to be applied to the manufacturing system.

Step 2. The Simulation model uses the control policy structure defined in the previous step as input for conducting experiments in order to evaluate the manufacturing system's performance. Hence, for given values of the control factors, the cost incurred is obtained.

Step 3. The Experimental design approach defines how the control factors can be varied in order to determine the effects of the main factors and their interactions (i.e., analysis of variance or ANOVA) on the cost through a minimal set of simulation experiments. 
Step 4. The Response surface methodology is then used to obtain the relationship between the cost incurred and significant main factors and interactions given in the previous step. The model obtained is then optimized in order to determine the best values of the factors known here as $Z_{i}^{*}$ for production. The Near-optimal control policy $u\left(Z_{i}^{*}\right)$ is an improved hedging point policy to be applied to the system. The application of the proposed control approach gives the production rates described by the equations by equations (16) to (18) for best values of factors $Z_{i}^{*}$.

\section{Simulation model}

The essence of the resolution approach is the simulation model developed using the Visual SLAM language (Pritsker and O'Reilly (1999)) and combines discrete-continuous changes describing the dynamics of the system presented in Figure 1 and governed by the production control policy developed in previous sections. This model consists of several networks and user C sub-routines, each of which describes a specific task in the system (i.e., control policy, states of the system, inventory control, etc.). The diagram of the simulation model is shown in Figure 4 with the following block descriptions:

1. The INITIALIZATION block sets the values of $Z_{i}(i=1,2)$, the demand rate $(d)$, and the machine parameters $\left(u_{1}^{\max }, u_{2}^{\max }, p_{1}, r_{1}\right)$, etc.). The maximum and minimum time step specifications for integration of the cumulative variables and allowable errors are also assigned at this step as well as the simulation time $T_{-}$fin and the time for the warm up period after which statistics are cleared.

2. The DEMAND RATE block performs the arrival of the demand for the production system at each $1 / \mathrm{d}$ unit of time. Verification is then performed on the inventory values. The inventory or the backlog level is then updated.

3. The $M_{1}$ block represents the central machine of the manufacturing cell. Due to its random availability (i.e., failure events and repair activities), $M_{1}$ capacity falls short to meet the demand $d$.

4. The $M_{2}$ block represents the reserve machine which is called to ensure a long term satisfaction of the demand. 


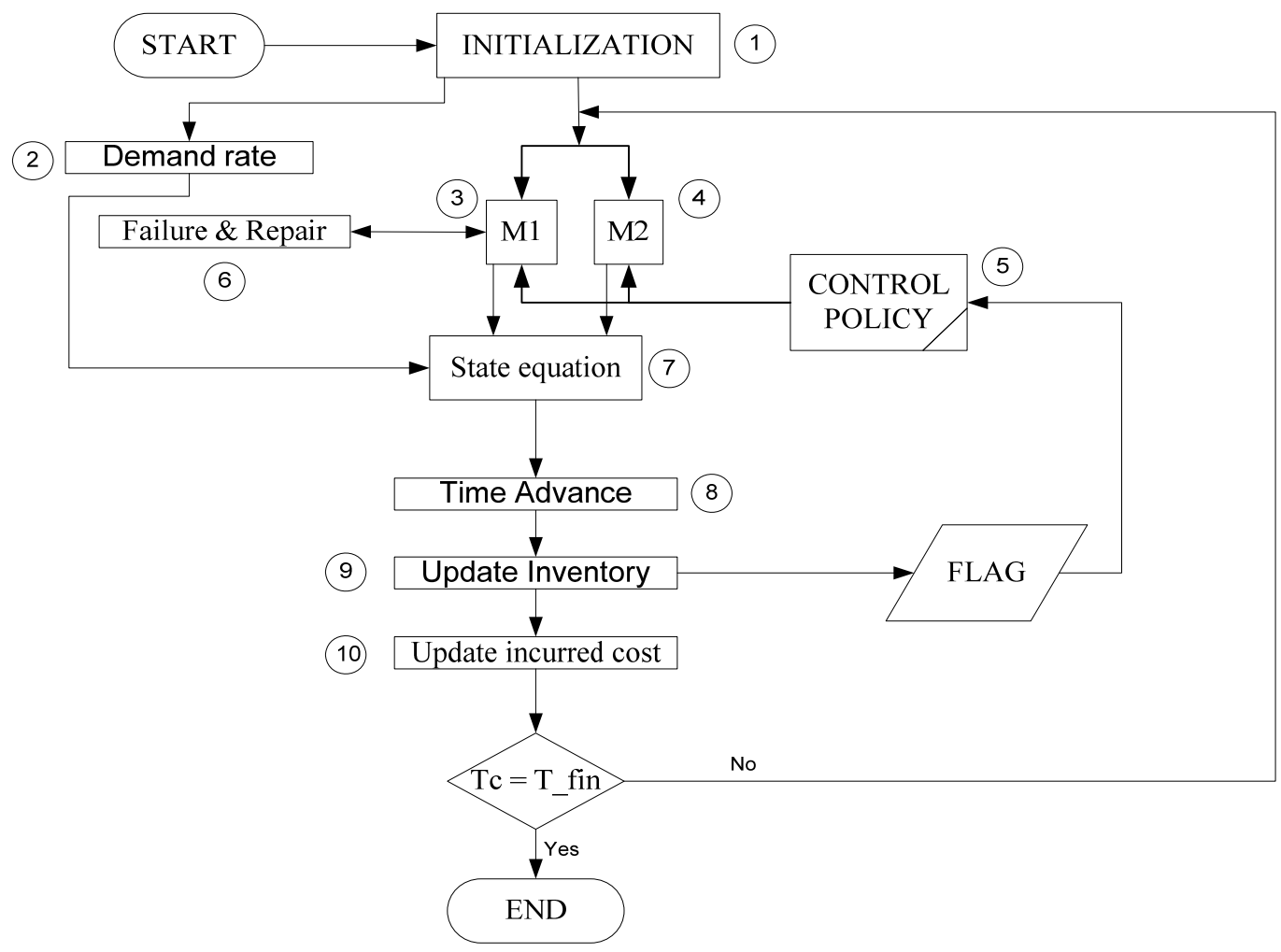

Figure 4. Diagram of simulation model

5. The CONTROL POLICY block is defined in the section 3 (see equations (16) to (18)) for the system production rates. The control policy is called by the output of the FLAG block. This block is used to permanently verify the variation in the surplus level $x(t)$. The production rate of $M_{1}$ is set to a zero value if $x(t)>Z_{1}$, otherwise it is either set to the demand rate $\left(u_{1}=d\right)$ if $x(t)=Z_{1}$ or to the maximum $\operatorname{rate}\left(u_{1}^{\max }\right)$ if $x(t)<Z_{1}$. The machine $M_{2}$ must produce at its maximum production rate $\left(u_{2}^{\max }\right)$ as long as the stock level $x(t)$ is lower or equal to the hedging level $Z_{2}$. Above $Z_{2}$ the machine is shut down and its production rate is fixed to zero.

6. The FAILURE \& REPAIR block samples the time to failure $\left(p_{1}^{-1}\right)$ and time to repair $\left(r_{1}^{-1}\right)$ of machine $M_{1}$ using probability distributions. When a failure arrives, $M_{1}$ is preempted until reparation is performed. The operational states of the machine $M_{1}$ is incorporated in the state equations by the means of logical variables multiplying the 
production rates. Demands are therefore not satisfied during that period unless the inventory is positive.

7. The STATE EQUATION is equation (1), defined as a $\mathrm{C}$ language insert. It describe the inventory and backlog variables using the production rates set by the control policy and the binary variables from the failure and repair of machine $M_{1}$.

8. The TIME ADVANCE block uses an algorithm provided by simulation software. It is a combination of discrete event scheduling (failures and repair), continuous variable threshold crossing events and time step specifications.

9. The UPDATE INVENTORY block traces the variation of the surplus level as machines produce or as demand arrival occurs (i.e. production either increases surplus, if there is no backorder, or it satisfies the cumulative demand, and hence decreases the backorder level).

10. The UPDATES INCURRED COST block calculates the incurred cost according to the levels of the inventory and backlog variables $\left(X^{+}\right.$and $\left.x^{-}\right)$, the unit costs $\left(C^{+}\right.$and $\left.C^{-}\right)$, which machine is producing $\left(M_{1}\right.$ and/or $\left.M_{2}\right)$ and their production costs $\left(C_{M_{1}}^{1}, C_{M_{1}}^{2}\right.$, and $C_{M_{2}}$ ).

\section{Experimental design and response surface methodology}

Given that an optimal solution of the stochastic control problem described in sections 2 exists and given the convexity property of the cost function, we define three levels for each factor $Z_{1}$ and $Z_{2}$ to obtain a convex estimated cost function. These observations give rise to a complete $3^{2}$ experimental design (Montgomery (2005)).

\subsection{Numerical example}

The following values of the operational and cost parameters, caracterizing the central and the reserve machines, are considered:

$d=100$ units $/ \mathrm{TU}, u_{1}^{\max }=125$ units/TU, $u_{2}^{\max }=25$ units/TU; $p_{1}^{-1}=0.25 \mathrm{TU}$ and $r_{1}^{-1}=0.1 \mathrm{TU}$;

$\mathrm{c}^{+}=10 \$ /$ unit/TU and $\mathrm{c}^{-}=100 \$ /$ unit/TU;

$C_{M_{1}}^{1}=20 \$$ /unit, $C_{M_{1}}^{2}=40 \$$ /unit, and $C_{M_{2}}=150 \$$ /unit. 
The minimum and the maximum values of $Z 1$ were first observed using simulation experiments. The independent variable levels were then chosen as presented in Table 3.

Table 3. Level of independent variables

\begin{tabular}{|c|c|c|c|}
\hline Factor & Low Level & Center & High Level \\
\hline $\mathbf{Z 1}$ & 40 & 60 & 80 \\
\hline $\mathbf{k}(\mathbf{Z} 2 / \mathbf{Z 1})$ & 0.05 & 0.5 & 0.95 \\
\hline
\end{tabular}

Five replications were conducted for each combination of the factors and a variance reduction technique called common random numbers (Law and Kelton (2000)) was used.

\subsection{Result Analysis}

The statistical analysis of the simulation data consists of the multifactor analysis of variance (ANOVA). This is done using a statistical software application (STATGRAPHICS) to provide the effects of the two independent variables $\left(Z_{1}\right.$, and $\left.\mathrm{k}\right)$ on the dependant variable (Total cost). The ANOVA table corresponding to the generated data is illustrated in Table 4. All p-values are less than $5 \%$. Consequently, we conclude that the main factors $Z_{1}$, and $k$, their quadratic effects, as well as their interactions are significant at the 0.05 level. The $R_{\text {adjusted }}^{2}$ value of 0.9744 from the ANOVA table states that more than $97 \%$ of the total variability is explained by the model (Montgomery (2005)).

Table 4. ANOVA table

\begin{tabular}{|c|c|c|c|c|c|}
\hline & Sum of squares & d.f. & Mean square & F-ratio & P-value \\
\hline$Z_{1}$ & 7977.89 & 1 & 7977.89 & 15.71 & $\mathbf{0 . 0 0 0 3}$ \\
\hline$k$ & 63809.9 & 1 & 63809.9 & 125.65 & $\mathbf{0 . 0 0 0 0}$ \\
\hline$Z_{1}^{2}$ & 7860.27 & 1 & 7860.27 & 15.48 & $\mathbf{0 . 0 0 0 4}$ \\
\hline$Z_{1} \times k$ & 106221. & 1 & 106221. & 209.17 & $\mathbf{0 . 0 0 0 0}$ \\
\hline$k^{2}$ & 577373. & 1 & 577373. & 1136.95 & $\mathbf{0 . 0 0 0 0}$ \\
\hline blocks & 4100.3 & 4 & 1025.07 & 2.02 & 0.1132 \\
\hline Total Error & 17773.9 & 35 & 507.826 & & \\
\hline Total (corr) & 785117. & 44 & & & $R_{\text {adjusted }}^{2}=\mathbf{0 . 9 7 4 4}$ \\
\hline
\end{tabular}

The residual analysis was used to verify the adequacy of the model. A residual versus predicted value plot and normal probability plot were used to test the homogeneity of the variances and the residual normality respectively. We conclude that the model is satisfactory. The second order model of the total cost is given by: 


$$
\text { Cost }=6461,77-11,6442 \times Z_{1}-1774,93 \times k+0,0700904 \times Z_{1}^{2}+8,09745 \times Z_{1} \times k+1186,6 \times k^{2}
$$

The near-optimal control policy to be applied to the considered manufacturing system is defined by the minimum of the cost function (19) located at $Z_{1}^{*}=49.66, k^{*}=0.578\left(Z_{2}^{*}=28.72\right)$ as shown in Figure 5. Cost ${ }^{*}=5659.31$ results from this control policy. $Z_{1}^{*}$ and $Z_{2}^{*}$ define the bestState Dependent Hedging Point Policy to be applied to the manufacturing system considered.

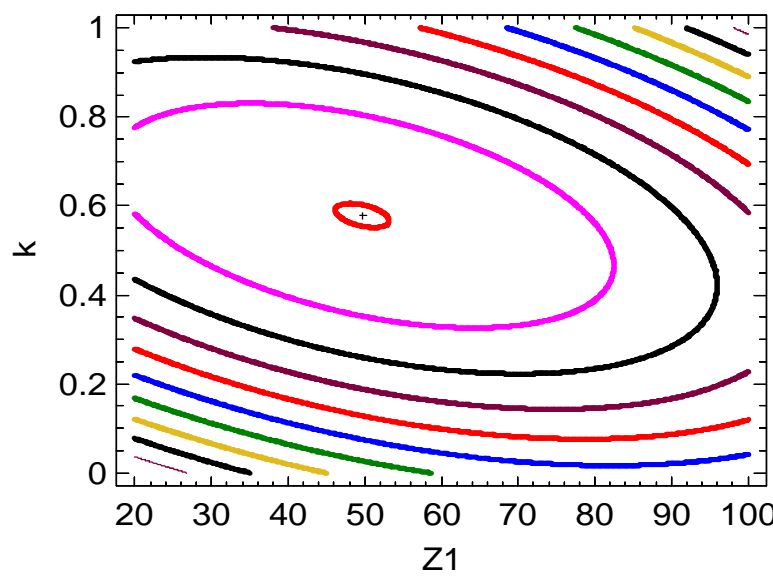

Figure 5. Contours of estimated Response Surface

\subsection{Sensitivity analysis}

A set of numerical examples are considered to measure the sensitivity of the obtained control policy with respect to inventory, backlog and production costs (i.e., $C^{+}$and $C^{-}, C_{M_{1}}^{1}$, $C_{M_{1}}^{2}$ and $C_{M_{2}}$ ). The following variations, illustrated in Table 5, are explored and compared to the basic case (experience No.1):

- Varying $C^{+}$: decreasing $C^{+}$(experience No.2) results in a tendency to increase the stock level in order to avoid further backlog costs $\left(\mathbf{Z}_{1}{ }^{*}\right.$ increases). Consequently, $\mathbf{M}_{1}$ needs more support from $\mathrm{M}_{2}$, which has to work more to guaranty the increasing of the stock level $\left(\mathbf{k}^{*}\right.$ increases). Increasing $C^{+}$gives the opposite results (experience No.3).

- Varying $C^{-}$: decreasing $C^{-}$(experience No.4) results in a tendency to decrease the stock level in order to avoid further inventory costs $\left(\mathbf{Z}_{1}{ }^{*}\right.$ decreases $)$. Consequently, $\mathrm{M}_{1}$ needs less support from $\mathrm{M}_{2}$, which has to work less to decrease its working cost ( $\mathbf{k}^{*}$ decreases). Increasing $C^{-}$gives the opposite results (experience No.5). 
Table 5. Sensitivity analysis table for the control Point policy

\begin{tabular}{|c|c|c|c|c|c|c|c|c|c|}
\hline No. & $C^{+}$ & $C^{-}$ & $C_{M_{1}}^{1}$ & $C_{M_{1}}^{2}$ & $C_{M_{2}}$ & $Z_{1}^{*}$ & $\begin{array}{l}\mathbf{Z}_{2}^{*} \\
\left(\mathbf{k}^{*}\right)\end{array}$ & $\operatorname{Cost}^{*}$ & Remark \\
\hline 1 & 10 & 100 & 20 & 40 & 200 & 64.12 & $\begin{array}{c}27.88 \\
(0.434)\end{array}$ & 6235.59 & Basic case \\
\hline 2 & 5 & 100 & 20 & 40 & 200 & 76.91 & $\begin{array}{r}37.84 \\
(0.492) \\
\end{array}$ & 6070.48 & $\begin{array}{l}\mathbf{Z}_{1}^{*} \text { increases } \\
\mathbf{k}^{*} \text { increases }\end{array}$ \\
\hline 3 & 15 & 100 & 20 & 40 & 200 & 52.15 & $\begin{array}{c}21.38 \\
(0.410)\end{array}$ & 6363.14 & $\begin{array}{l}\mathbf{Z}_{1}{ }^{*} \text { decreases } \\
\mathbf{k}^{*} \text { decreases }\end{array}$ \\
\hline 4 & 10 & 50 & 20 & 40 & 200 & 60.90 & $\begin{array}{c}16.73 \\
(0.275)\end{array}$ & 6097.22 & $\begin{array}{c}\mathbf{Z}_{1}{ }^{*} \text { decreases } \\
\mathbf{k}^{*} \text { decreases }\end{array}$ \\
\hline 5 & 10 & 150 & 20 & 40 & 200 & 68.45 & $\begin{array}{c}35.78 \\
(0.523)\end{array}$ & 6317.78 & $\begin{array}{l}\mathbf{Z}_{1}^{*} \text { increases } \\
\mathbf{k}^{*} \text { increases }\end{array}$ \\
\hline 6 & 10 & 100 & 10 & 40 & 200 & 53.77 & $\begin{array}{c}28.87 \\
(0.537)\end{array}$ & 6206.95 & $\begin{array}{l}\mathbf{Z}_{1}^{*} \text { decreases } \\
\mathbf{k}^{*} \text { increases }\end{array}$ \\
\hline 7 & 10 & 100 & 30 & 40 & 200 & 69.70 & $\begin{array}{l}26.63 \\
(0.382)\end{array}$ & 6244.92 & $\begin{array}{l}\mathbf{Z}_{1}^{*} \text { increases } \\
\mathbf{k}^{*} \text { decreases }\end{array}$ \\
\hline 8 & 10 & 100 & 20 & 30 & 200 & 70.69 & $\begin{array}{c}36.34 \\
(0.373)\end{array}$ & 5352.56 & $\begin{array}{l}\mathbf{Z}_{1}{ }^{*} \text { increases } \\
\mathbf{k}^{*} \text { decreases }\end{array}$ \\
\hline 9 & 10 & 100 & 20 & 50 & 200 & 49.65 & $\begin{array}{c}26.70 \\
(0.579)\end{array}$ & 7086.52 & $\begin{array}{l}\mathbf{Z}_{1}^{*} \text { decreases } \\
\mathbf{k}^{*} \text { decreases }\end{array}$ \\
\hline 10 & 10 & 100 & 20 & 40 & 150 & 49.66 & $\begin{array}{c}28.72 \\
(0.578)\end{array}$ & 5659.31 & $\begin{array}{c}\mathbf{Z}_{1}{ }^{*} \text { decreases } \\
\mathbf{k}^{*} \text { increases }\end{array}$ \\
\hline 11 & 10 & 100 & 20 & 40 & 250 & 70.69 & $\begin{array}{l}26.30 \\
(0.373)\end{array}$ & 6779.77 & $\begin{array}{l}\mathbf{Z}_{1}^{*} \text { increases } \\
\mathbf{k}^{*} \text { decreases }\end{array}$ \\
\hline
\end{tabular}

- Varying $C_{M_{1}}^{1}$ : decreasing $C_{M_{1}}^{1}$ (experience No.6) results in a tendency to work more at the demand rate (system spends more time in area 3 (fig.3); stock level $=Z_{1}$ ). Consequently, $\mathbf{Z}_{1}{ }^{*}$ should decreases and $\mathrm{M}_{1}$ should get more support from $\mathrm{M}_{2}\left(\mathbf{k}^{*}\right.$ increases). Increasing $C_{M_{1}}^{1}$ gives the opposite results (experience No.7).

- $\quad$ Varying $C_{M_{1}}^{2}$ : decreasing $C_{M_{1}}^{2}$ (experience No.8) results in a tendency to make machine $\mathrm{M}_{1}$ work more at the maximum rate $\left(u_{1}^{\max }\right)$ (system spends more time in area 2 (fig.3)). Consequently, $\mathrm{M}_{1}$ should get less support from $\mathrm{M}_{2}\left(\mathbf{k}^{*}\right.$ decreases) and in order to avoid further backlog costs $\mathbf{Z}_{1}{ }^{*}$ should increases. Increasing $C_{M_{1}}^{2}$ gives the opposite results (experience No.9).

- Varying $C_{M_{2}}$ : decreasing $C_{M_{2}}$ (experience No.10) results in a tendency to make machine $\mathrm{M}_{2}$ work more (system spends more time in area 1 (fig.3)). Consequently, $\mathbf{k}^{*}$ 
increases and the system is more feasible and this results in a tendency to decrease the stock level in order to avoid further inventory costs $\left(\mathbf{Z}_{1}{ }^{*}\right.$ decreases $)$. Increasing $C_{M_{2}}$ gives the opposite results (experience No.11).

It clearly appears that the results obtained make sense and confirm the numerical observation in the sense that when a cost decreases (resp. increases), the area where this costs is incurred increases (resp. decreases).

\section{Comparison between SDHPP policy and the classical redundant structure policies}

In this section, the SDHPP policy is compared first to a control policy based on classical stand-by operational structure (passive redundancy) where the manufacturing cell is composed of a central machine and a stand-by one called upon in support if the central machine breaks down and until reparation ends. If we consider the classical stand-by based control policy, we notice that it is not feasible. In fact, after transforming equation (6), the manufacturing cell production capacity equals 96.43 units/TU (Eq. 20), which is less than the demand rate of 100 units/TU. Consequently, and under the classical stand-by based control policy, the demand will never be satisfied. Using our system's data, we obtain:

$$
u_{1}^{\max } \times \frac{r_{1}}{r_{1}+p_{1}}+u_{2}^{\max } \times\left(1-\frac{r_{1}}{r_{1}+p_{1}}\right)=96.43
$$

The SDHPP policy has also been compared to a control policy based on parallel operational structure (active redundancy) where the manufacturing cell is composed of two parallel machines. This policy has been considered by Gharbi and Kenne (2003). Using the data as the basic case of table 5, the estimated second-order model of the total cost incurred by such a policy is given by:

$$
\text { Cost- parallel }=7028.9-15.7547 * Z_{1}+0.200683 * Z_{1}^{2}
$$

The near-optimal control policy to be applied to the considered manufacturing cell is defined by the minimum of the cost function (21) located at $Z_{1}^{*}=39.25$. The Cost-pallel* $=6719.70$ resulting from this control policy is significantly greater than that obtained by our proposed policy $($ Cost $*=6235.59)$ involving a relative cost reduction of $7.76 \%$. 


\section{Extension to manufacturing system with non-exponential failure and repair time distributions}

In the literature of control theory, no satisfactory method has been devised for the stochastic optimal control of manufacturing systems subject to non-exponential machine up and down times. In fact, the exponential distribution is used to develop optimality conditions, as shown in section 2. With non-exponential failure and repair time distributions, optimality conditions are very difficult to develop. However, such a situation is usually encountered in real manufacturing systems. We refer the reader to Law and Kelton (2000), for details on commonly used failure and repair time probability distributions.

Despite the types of failure and repair time distributions, a near-optimal control policy could be determined in a much more complex situation (with non-exponential up and down distribution times for machines). Using the same illustrative example presented in section 6.1, we will show that the proposed approach is efficient and robust when considering general time to failure and time to repair distributions of machine $M_{1}$. To deal with extensions involving the manufacturing system size and its stochastic processes, we refer the reader to Gharbi et al. (2006) where optimal control theory is combined to simulation modelling to find the best production strategy for multiple-machine multiple-product systems with setups. Note that, in this context, Gharbi et al. (2006) have shown that the developed production and setup policy and which is based on the class of MHP policy guarantees better and more realistic results.

Without loss of generality, we use a lognormal time distribution as it is often encountered in real maintenance of manufacturing systems (Law and Kelton (2000)). For purposes of homogeneity with the exponential distribution, we assume that the mean and standard deviation are the same. Our experimental results show that the total cost function can also be represented by a convex function under non-exponential time to failure and time to repair distributions. Using the same data as in section 6.1, the estimated second-order model for lognormal distribution is given by:

Cost_LN $=6386,78-10,023 \times Z_{1}-1640,6 \times k+0,0594 \times Z_{1}^{2}+7,7475 \times Z_{1} \times k+1111,52 \times k^{2}$

The $R_{\text {adjusted }}^{2}$ value of 0.9792 from the ANOVA table states that more than $97 \%$ of the total variability is explained by the model. The near-optimal control policy to be applied to the manufacturing system considered is defined by the minimum of the cost function (22) located at $Z_{1}^{*}=46.94, k^{*}=0.574\left(Z_{2}^{*}=26.94\right)$. The Cost_LN*= 5680.32 resulting from this control policy 
is very close to the one obtained under exponential time to failure and time to repair distributions of machine $M_{1}$ (Cost_Expon*= 5659.31).

\section{Conclusions}

In this paper, we studied the production control problem for a mono-product manufacturing cell comprising an unreliable central machine with adjustable capacity. In order to respond to the demand, a reserve machine is called upon in support by allowing capacity adjustment. The production control problem was formulated as a continuous time dynamic programming problem and HJB equations were derived. Numerical approach was also proposed to solve the HJB equations of the problem and to obtain near-optimal production control policy.

The optimal control policy has been shown to be described by a State Dependent Hedging Point Policy (SDHPP) with two hedging inventory levels. The SDHPP policy consists at operating the two machines at their maximum production rates until they reach the first hedging level. At this threshold, the reserve machine is stopped and the central one continues producing until reaching a second hedging level. At the second threshold, the central machine reduces its production rate to that of the demand, and maintaining the inventory at its maximum level. If a breakdown occurs, and the inventory level decreases below the first hedging level, the reserve machine starts producing at its maximum production rate.

Based on the obtained numerical solution, a parameterized near-optimal control policy was derived. Such a policy depends on the stock threshold levels. To determine the parameters of the control policy, and hence, to achieve a close approximation of the optimal production policy, an experimental approach based on design of experiment, simulation modeling and response surface methodology has been proposed. To outline the robustness of the developed policy facing expected variations of the system parameters several sensitivity analysis were conducted.

The proposed SDHPP policy has also been compared to production control policies based on classical redundant structure (stand-by and parallel machines). The results show that the proposed policy outperforms classical ones.

The proposed approach offers an easily applied procedure to tackle realistic manufacturing systems situations. This issue is outlined by extending the study to cover manufacturing systems facing non-exponential failure and repair time distributions. 


\section{References}

1. Akella, R. and Kumar, P. R. «Optimal control of production rate in a failure-prone manufacturing system». IEEE Transactions on Automatic Control, Vol.AC-31, N0 2, pp: 116-126, 1986.

2. Andrés, C., Albarracín, J. M., Tormo, G., Vicens, E., García-Sabater, J. P. «Group technology in a hybrid flowshop environment: A case study». European Journal of Operational Research, Vol. 167, No. 1, pp. 272-281, 2005.

3. Askin, R.G. and Standridge, C.R. «Modeling and Analysis of Manufacturing Systems", John Wiley \& Sons, Inc., New York, 1993.

4. Berthaut, F., Gharbi, A., and Pellerin, R., «Joint hybrid repair and remanufacturing systems and supply control ». International Journal of Production Research, online 16 June 2009.

5. Bielecki, T. R., and P. R. Kumar, «Optimality of zero-inventory policies for unreliable manufacturing systems ». Operations Res., vol. 36, no. 4, pp. 532-541, 1988.

6. Burbidge, J.L. «Change to Groupe Technology: Process Organization is Obsolete». International Journal of Production Research, Vol. 30, No. 5, pp. 1209-1219, 1992.

7. Dehayem Nodem, F.I., Kenne, J.P., and Gharbi, A. «Hierarchical decision making in production and repair/replacement planning with imperfect repairs under uncertainties ». European Journal of Operational Research, Vol. 198, pp. 173-189, 2009.

8. Freiheit, T., Shpitalni, M., Hu, S.J., and Koren, Y. «Productivity of synchronized serial production lines with flexible reserve capacity ». International Journal of Production Research, Vol. 42, pp. 2009-2027, 2004.

9. Gershwin, S. B. « Design and operation of manufacturing systems: the control-point policy». IIE Transactions, Vol 32, pp 891-906. 2000.

10. Gharbi, A. and Kenne J.P. «Optimal production control problem in stochastic multipleproduct multiple-machine manufacturing systems», IIE Transactions, Vol.35, N0.10, pp.941-952. 2003.

11. Gharbi A., Kenne J. P. and Hajji A. «Operational Level-Based Policies in Production rate control of unreliable manufacturing systems with setups». International Journal of Production Research, Vol. 44, No. 3, pp. 545-567, 2006.

12. Gharbi, A., Pellerin, R., and Sadr, J. «Produciton rate control for stochastic remanufacturing systems ». International Journal of Production Economics, Vol. 112, pp. $37-47,2008$. 
13. Hajji A., Gharbi A. and Kenne J. P. « Joint replenishment and manufacturing activities control in two stages unreliable supply chain ». International Journal of Production Research, Vol. 47, No. 12, pp. 3231-3251, 2009.

14. Jardine, A.K.S., and Tsang, A.H.C. « Maintenance, Replacement, and Reliability: Theory and Applications ». CRC Press, London, 2005.

15. Kenne, J.P., Boukas, E.K., and Gharbi, A. «Control of Production and Corrective Maintenance rates in a Multiple-Machine, Multiple-Product Manufacturing System ». Mathematical and Computer Modelling, Vol. 38, pp. 351-365, 2003.

16. Kenne J.P., Gharbi, A., et Beit, M. «Age-Dependent Production Planning and Maintenance Strategies in Unreliable Manufacturing Systems with Lost Sale». European Journal of Operational Research. Vol. 178 pp. 408-420, 2007.

17. Kimemia, J. G. and Gershwin, S. B. «An algorithm for the computer control production in flexible manufacturing systems». IIE Transactions, Vol 15, pp 353-362. 1983.

18. Koren, Y., Jovane, F., Heisel, U., Moriwaki, T., Pritschow, G., Ulsoy, G., and VanBrussel, H. «Reconfigurable Manufacturing Systems: A Keynote paper». CIRP Annals, Vol. 48, No. 2, pp. 6-12, 1999.

19. Koren, Y. and Ulsoy, G. «Reconfigurable Manufacturing System Having a Method for Changing its Production Capacity». US patent \# 6,349,237; issue date: 2/19/2002.

20. Kushner, H. J. and Dupuis, P. G. «Numerical methods for stochastic control problems in continuous time», springer-verlag, New York,1992.

21. Law, A. M. and Kelton, W. D. «Simulation modeling and analysis», McGraw-Hill, New York, 2000.

22. Liberopoulos, G., and Caramanis, M. «Production control of manufacturing systems with production rate-dependent failure rates». IEEE Trans. Automat. Contr., vol. 39, pp. 889895, 1994.

23. Liberopoulos, G., and $\mathrm{Hu}$, J. Q. «On the ordering of hedging points in a class of manufacturing flow control models». IEEE Trans. Automat. Contr., vol. 40, pp. 282-286, 1995.

24. Montgomery, D. C. «Design and analysis of experiments», John Wiley \& Sons, New York, 2005.

25. Pellerin, R., Boulet, J.F., Gharbi, A., and Kenne, J.P., « Développement d'une politique intégrée de contrôle des taux de production et de maintenance corrective avec diagnostic », INFOR, Vol. 45, No. 4, pp. 197-207, 2007. 
26. Pritsker, A. A. B. and O'reilly, J. J. «Simulation with Visual SLAM and Awesim». John Wiley \& Sons, New York, 1999.

27. Sethi, S. P., Yan, H., Zhang, H. and Zhang, Q. « Optimal and Hierarchical Controls in Dynamic Stochastic Manufacturing Systems: A Survey». Manufacturing \& Service Operations Management, Vol. 4, No. 2, pp. 133-170, 2002.

28. Sharifnia, A. «Production control of a manufacturing system with multiple machine states». IEEE Transactions on Automatic Control, Vol. 33, N0 7, pp: 620-625, 1988.

29. Wu, X., Chu, C.-H., Wang, Y., Yan, W. «A genetic algorithm for cellular manufacturing design and layout». European Journal of Operational Research, Vol. 181, No. 1, pp. 156-167, 2007.

30. Yang, M.-S., Yang, J.-H. «Machine-part cell formation in group technology using a modified ART1 method». European Journal of Operational Research, Vol. 188, No. 1, pp. 140-152, 2008.

31. Zanoni, S., Ferretti, I., and Tang, O. «Cost performance and bullwhip effect in a hybrid manufacturing and remanufacturing system with different control policies». International Journal of Production Research, Vol. 44, No. (18-19), pp. 3847-3862, 2006. 